\title{
ACTIVITE PHOTOSYNTHETIQUE DU PHYTOPLANCTON DANS LE BARRAGE HYDROELECTRIQUE D'AYAME I (CÔTE D'IVOIRE)
}

\author{
A. OUATTARA' ${ }^{1}$, G. GOURENE ${ }^{1}$ et N. PODOOR ${ }^{2}$ \\ 'Laboratoire d'Environnement et de Biologie Aquatique, Université d'Abobo-Adjamé 02 BP 801 Abidjan 02, \\ Côte d'Ivoire. E.mail : allassane_ouattara@hotmail.com \\ ${ }^{2}$ Katholieke Universiteit Leuven, Instituut voor Plantkunde, Morphologie, Systematiek en Ecologie der planten, \\ Kardinaal Mercierlaan 92, B-3001 Leuven, Belgique.
}

\begin{abstract}
RESUME
L'activité photosynthétique du phytoplancton du barrage hydroélectrique d'Ayamé I a été suivie au cours de six campagnes de mesures sur deux stations (Bakro, Ayamé) afin d'évaluer son évolution spatio-temporelle et d'identifier les variables qui la contrôlent. Les mesures de la productivité photosynthétique du phytoplancton ont été effectuées dans la couche euphotique (0-3 m). Les résultats montrent une variabilité temporelle de l'activité photosynthétique dans les deux stations du lac. Les productivités phytoplanctoniques sont maximales à Bakro et à Ayamé en début de saison des pluies (respectivement 0,35 et $0,81 \mathrm{mg} \cdot \mathrm{C} \cdot \mathrm{m}^{-2} \cdot \mathrm{j}^{-1}$ ) et minimales en saison sèche (respectivement 0,18 et $0,43 \mathrm{mg} \cdot \mathrm{C} \cdot \mathrm{m}^{-2} \cdot \mathrm{j}^{-1}$ ). Les productivités phytoplanctoniques ont été positivement corrélées aux nitrates $(r>0,8 ; p<0,05)$ et aux phosphates $(r>0,6 ; p<0,05)$, ce qui souligne l'importance des nutriments dans la productivité du phytoplancton.
\end{abstract}

Mots clés : Activité photosynthétique, phytoplancton, barrage hydroélectrique, Côte d'Ivoire.

\section{ABSTRACT}

PHOTOSYNTHETIC ACTIVITY OF PHYTOPLANKTON IN THE HYDROELECTRIC RESERVOIR OF AYAME I (CÔTED'IVOIRE)

Photosynthetic activity of phytoplankton in the hydroelectric reservoir of Ayamé I was studied during 6 data acquisition campaigns at two stations (Bakro, Ayamé). The main objective was to monitor spatial and temporal changes in phytoplankton productivity and identifiy potential variables responsible for the changes. Phytoplankton photosynthetic activity was determined in the euphotic layers $(0-3 \mathrm{~m})$ of the lake. Results show changes in photosynthetic activity with time in the two stations. Maximum concentrations of carbon assimilation were observed during the begining of the rainy season (0.35 and $\left.0.81 \mathrm{mg} . \mathrm{C}_{\mathrm{m}} \mathrm{m}^{-2} . \mathrm{j}^{-1}\right)$ and minimum activity during the dry season (0.18 and $\left.0.43 \mathrm{mg} . \mathrm{C}_{\mathrm{m}} \mathrm{m}^{-2} \cdot \mathrm{j}^{-1}\right)$ at Bakro and Ayamé, respectively. The phytoplankton productivities correlated with nitrate $(r>0.8 ; p<0.05)$ and phosphorus levels $(r>0.6 ; p<0.05)$ which underscores the importance of nutrients in phytoplankton productivity.

Key words : Photosynthetic activity, phytoplankton, hydroelectric reservoir, Côte d'Ivoire.

\section{INTRODUCTION}

Les producteurs primaires (phytoplancton, périphyton, macrophytes) sont des communautés déterminantes dans le fonctionnement d'un écosystème aquatique. Ils sont à l'origine de la matière organique élaborée à partir des éléments minéraux du milieu, contribuant à la mise en place des chaînes trophiques (Vaquer et al., 1997).

La retenue d'eau d'Ayamé l est un réservoir créé en zone forestière tropicale destiné à la production d'électricité. Cette situation a permis la colonisation et l'implantation dans ce lac d'un peuplement phytoplanctonique à partir d'une rivière aménagée (la rivière Bia) peu favorable, à 
l'origine, à son développement (Ouattara et al., 2001). Ce plancton végétal y assure essentiellement la production primaire. Cette ressource trophique est exploitée notamment par la microfaune planctonique et par certains poissons du lac. Ces derniers constituent dans cet écosystème lacustre une ressource économique importante (Gourène et al., 1999).

Les travaux portant sur les estimations des productions primaires dans les lacs africains restent fragmentaires en ce qui concerne les échelles d'espace et de temps (Ganf, 1975 ; Kassoum, 1977 ; Degnbol et Mapila, 1982 ; Patterson et Kachinjika, 1995). Pourtant, de nombreux travaux se sont attachés à établir une relation significative entre la productivité primaire et les rendements de poisson dans les lacs (Melack, 1976 ; Oglesby, 1977 ; Biro et Voros, 1990). II a été notamment mis en évidence que la productivité primaire représente un meilleur estimateur de la productivité potentielle en poisson des lacs (Downing et al., 1990 ; Cyr et Peters, 1996). Ainsi, l'acquisition d'une meilleure connaissance de la base des réseaux trophiques dans les systèmes lacustres africains est-elle apparue nécessaire. Ce travail vise l'évaluation et le suivi spatio-temporel de l'activité photosynthétique du phytoplancton dans le lac d'Ayamé I.

\section{MATERIEL ET METHODES}

\section{CARACTERISTIQUES DU SITE D'ETUDE}

Le barrage hydroélectrique d'Ayamé I créé en 1959 , est situé entre $5^{\circ} 30^{\prime} \mathrm{N}$ et $3^{\circ} \mathrm{W}$. Il occupe une dépression allongée dans l'axe Nord-Sud longue de $80 \mathrm{~km}$ et large de $27 \mathrm{~km}$ en moyenne. Le niveau maximal de la retenue est de $30 \mathrm{~m}$ correspondant à une surface du plan d'eau de $190 \mathrm{~km}^{2}$ et un volume de $10^{9} \mathrm{~m}^{3}$. II est principalement alimenté par la rivière $\mathrm{Bia}$. $\mathrm{La}$ crête de développement $575 \mathrm{~m}$, est à la côte $88 \mathrm{~m}$ avec une hauteur sur fondation de $30 \mathrm{~m}$ (source : Compagnie Ivoirienne d'Electricité). Ce barrage alimente une seconde retenue (Ayamé II), $4 \mathrm{~km}$ en aval (Figure 1). La zone d'étude se trouve en milieu forestier ; le climat est caractérisé par deux saisons sèches (décembre-mars et août-septembre) et deux saisons pluvieuses (avril-juillet et octobrenovembre).

Six campagnes mensuelles de mesures (de décembre 1996 à mai 1997) ont été effectuées en deux points du lac d'Ayamé I :

- Ayamé $\left(5^{\circ} 36^{\prime} \mathrm{N}\right.$ et $\left.3^{\circ} 10^{\prime} \mathrm{W}\right)$, près de la digue ;

- Bakro ( $5^{\circ} 33^{\prime} \mathrm{N}$ et $\left.3^{\circ} 15^{\prime} \mathrm{W}\right), 20 \mathrm{~km}$ plus à l'ouest.

Au cours de ces campagnes, l'activité photosynthétique du phytoplancton et les caractéristiques physico-chimiques ont été mesurées.

\section{MESURE DES PARAMETRES PHYSICO- CHIMIQUES}

Les caractéristiques physico-chimiques de l'eau ont été mesurées directement, in situ, à l'aide d'appareils portatifs spécifiques (entre $8 \mathrm{~h}$ et $9 \mathrm{~h}$ ). La mesure de l'oxygène dissous a été faite à partir d'un oxymètre $96 \mathrm{WTW}$, le pH et la température à l'aide d'un $\mathrm{pH}$-mètre WTW. Enfin, ont également été mesurés la conductivité au moyen d'un conductimètre LF 92 WT et la transparence grâce au disque de Secchi. Les ions (orthophosphates, nitrates) ont été analysés par méthode colorimétrique à l'aide du spectrophotomètre HACH/DR -2000 sur des échantillons prélevés en surface.

\section{METHODE DE MESURE DE LA PRODUCTIVITE PRIMAIRE}

La production primaire a été estimée par la méthode de l'oxygène produit lors de la photosynthèse (Vollenweider, 1974). Elle consiste à isoler pendant un temps déterminé, une partie de la communauté planctonique dans des flacons transparents et une autre dans des flacons rendus opaques et replacés à la profondeur où les prélèvements ont été effectués à l'aide d'une bouteille de Van Dorn. La mesure de la quantité d'oxygène dissous dans l'eau de ces flacons, avant et après réimmersion, permet de déterminer la quantité d'oxygène produite par photosynthèse, aux profondeurs : $0 ; 1 ; 2$ et $3 \mathrm{~m}$. 


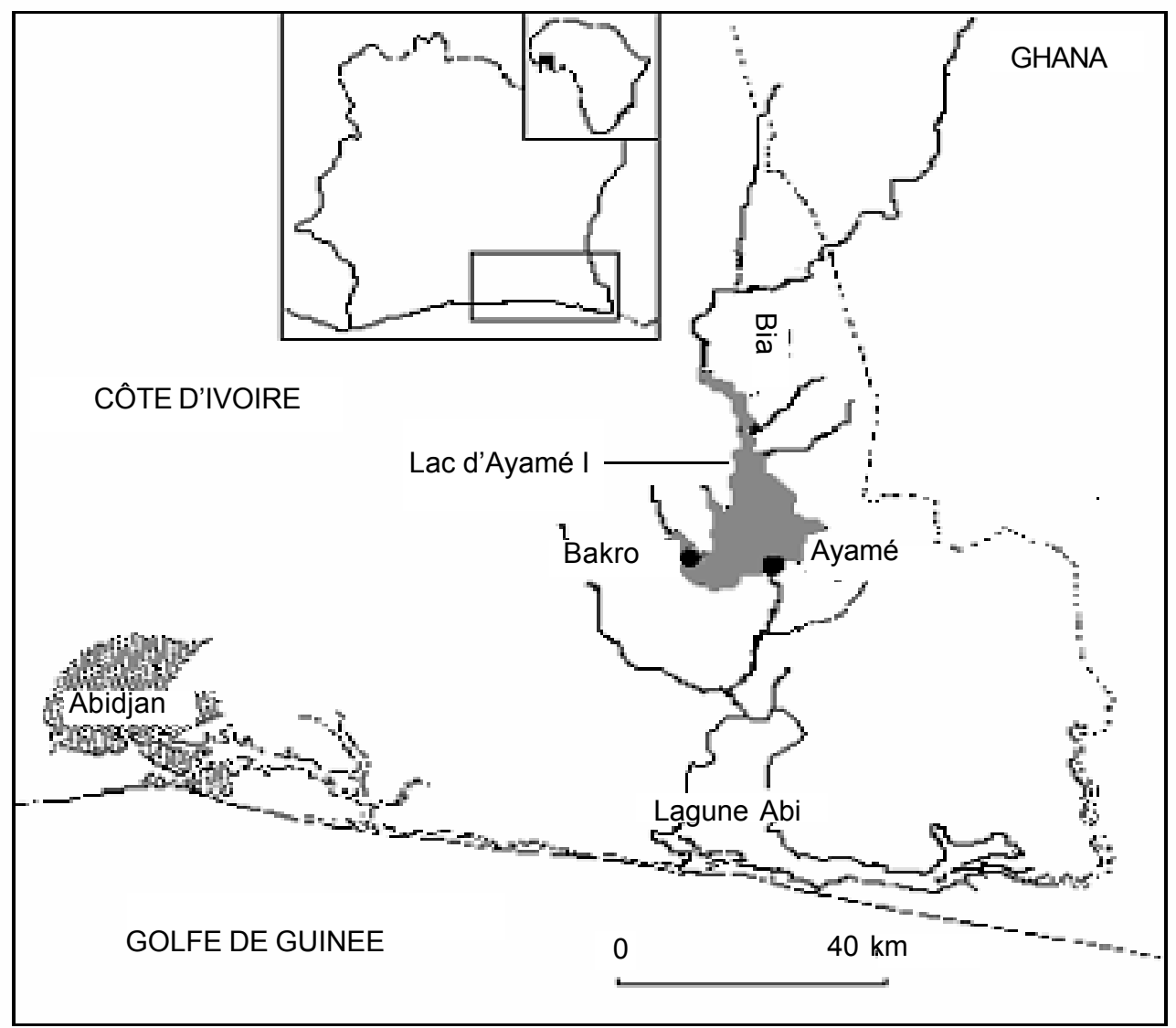

Figure 1 : Localisation des stations de mesures sur le lac d'Ayamé I. (Gourène et al., 1999) Location of sampling stations in the Ayamé I lake.

Deux (2) bouteilles en verres de $250 \mathrm{ml}$ chacune (une transparente et une opaque) ont été immergées pendant $4 \mathrm{~h}(10 \mathrm{~h} 30$ à $14 \mathrm{~h} 30 \mathrm{mn}$ ) à chaque profondeur. Cette tranche horaire a été retenue sur la base des travaux de Vollenweider (1974) et de Wetzel et Likens (1995) qui ont pu montrer que la production primaire horaire moyenne obtenue après une incubation d'échantillons d'eau de 3 à $4 \mathrm{~h}$ in situ, de part et d'autre du midi solaire, est représentative de l'activité photosynthétique journalière. Ainsi, en multipliant cette production primaire horaire moyenne par la durée d'insolation par jour (12 h en moyenne) l'on obtient la production primaire journalière. La production primaire correspondant à l'activité photosynthétique a été calculée, pour chaque profondeur d'incubation, de la façon suivante :

$a=$ quantité d'oxygène dissous dans l'eau avant l'incubation ;

$b$ = quantité d'oxygène dissous dans la bouteille claire après incubation ; $c=$ quantité d'oxygène dissous dans la bouteille opaque après incubation ;

$(a-c)=$ consommation d'oxygène par respiration;

$(b-a)=$ production nette en oxygène ;

$(b-a)+(a-c)=$ production brute en oxygène .

Dans cette étude, nous n'avons tenu seulement compte que de la production nette en oxygène du phytoplancton, car constituant le taux de production effectivement mis à la disposition des prédateurs phytoplanctonophages, ignorant ainsi les problèmes de photorespiration.

Les taux d'oxygène dissous ont été convertis en valeurs de carbone en les multipliant par un facteur de 0,375 (12 mg C/32 $\mathrm{mg} \mathrm{O}_{2}=0,375$ selon Wetzel et Likens, 1995).

Les données en unité de volume, exprimées en $\mu \mathrm{g} \mathrm{C} . \mathrm{I}^{-1} . \mathrm{j}^{-1}$, ont été converties en $\mathrm{mg} \mathrm{C} . \mathrm{m}^{-3} . \mathrm{j}^{-1}$ $\left(1 \mu \mathrm{g} . \mathrm{I}^{-1}=10^{-3} \mathrm{mg} \cdot \mathrm{I}^{-1}=1 \mathrm{mg} \cdot \mathrm{m}^{-3}\right)$. L'estimation de la production photosynthétique par unité 
de surface $\left(m g . C \cdot m^{-2} \cdot j^{-1}\right)$ requiert d'abord le tracé d'un profil vertical des valeurs de productivité photosynthétique par unité de volume (mg.C. $\mathrm{m}^{-3} \cdot \mathrm{j}^{-1}$ ) en fonction de la profondeur $(\mathrm{m})$. Ensuite, l'on fait une intégration du profil vertical obtenu pour avoir la productivité photosynthétique par unité de surface (Vollenweider, 1974 ; Wetzel et Likens, 1995).

\section{ANALYSE STATISTIQUE}

Afin d'analyser l'influence de l'environnement physico-chimique sur l'activité photosynthétique du phytoplancton à chaque station, une série de corrélations de Pearson a été réalisée. Les données de productivité phytoplanctonique ont été soumises à des analyses de variance (STATISTICA version 5.0) entre stations de mesure, en vue de déterminer l'importance des hétérogénéités spatiales des valeurs obtenues.

\section{RESULTATS}

\section{CARACTERISTIQUES PHYSICO-CHIMIQUES}

Le tableau 1 indique les résultats des analyses physico-chimiques effectuées sur les deux stations du lac d'Ayamé l. Les valeurs moyennes, minimales, maximales et les écart-types des paramètres mesurés sur chaque station y sont présentées. Les valeurs de température, de $\mathrm{pH}$ et de transparence de l'eau sont, de même ordre de grandeur à Bakro et à Ayamé. Les eaux sont relativement chaudes (température $>28^{\circ} \mathrm{C}$ ), proches de la neutralité $(\mathrm{pH}$ voisin de 7 ) et peu transparentes (transparence légèrement supérieure à $1 \mathrm{~m}$ ). Quant aux teneurs en $\mathrm{NO}_{3}-\mathrm{N}$, elles varient de 0,1 à $0,4 \mathrm{mg} / \mathrm{l}$ à Bakro et de 0,1 à $0,5 \mathrm{mg} / \mathrm{l}$ à Ayamé. En revanche, les concentrations en oxygène, sont inférieures à Bakro $(3,2$ à $6,7 \mathrm{mg} / \mathrm{l})$ qu' à Ayamé (5,2 à 7,8 mg/l). De même, les valeurs de conductivité et de $\mathrm{PO}_{4}-\mathrm{P}$, sont présentes à Bakro à des concentrations inférieures (respectivement de 55,7 à $65,9 \mu \mathrm{S} / \mathrm{cm}$ et de 0,5 à $0,9 \mathrm{mg} / \mathrm{l}$ ) à celles d'Ayamé (respectivement de 78 à $99,9 \mu \mathrm{S} / \mathrm{cm}$ et de 0,8 à $1,9 \mathrm{mg} / \mathrm{l})$. Les maximums ont été enregistrés en début de saison de pluies (avril-mai) tandis que les minimums ont été observés en saison sèche (janvier-février).

\section{VARIATIONS DE L'ACTIVITE PHOTOSYN- THETIQUE DU PHYTOPLANCTON}

Les mesures de production d'oxygène et d'assimilation de carbone concernant chacune des six campagnes sont détaillées dans le tableau 2. Les valeurs sont beaucoup plus faibles à Bakro qu'à Ayamé. Les mesures ont montré que le phytoplancton pouvait produire entre 11,1 et $108,9 \mu \mathrm{g} . \mathrm{I}^{-1} \cdot \mathrm{h}^{-1}$ d'oxygène à Bakro et entre 42,2 et $266,7 \mu \mathrm{g} \cdot \mathrm{I}^{-1} \cdot \mathrm{h}^{-1}$ d'oxygène à Ayamé, correspondant respectivement à une assimilation de carbone de 4,2 à 40,8 $\mu \mathrm{g} \cdot \mathrm{I}^{-1} \cdot \mathrm{h}^{-1}$ et de 15,8 à $100 \mu \mathrm{g} \cdot \mathrm{I}^{-1} \cdot \mathrm{h}^{-1}$. La répartition verticale de l'activité photosynthétique du phytoplancton sur l'ensemble de la période d'étude est représentée sur la figure 2. De décembre à février, les profils de production sont relativement

Tableau 1 : Valeurs minimales (Min), maximales (Max), moyennes (Moy) et écart-types (E-types) des différents paramètres physico-chimiques mesurés à chaque station.

Minimum (Min), maximum (Max), means (Moy) values with standard deviation for different physical and chemical parameters analysed at each station.

\begin{tabular}{lcccccccc}
\hline \multirow{2}{*}{ Paramètres } & \multicolumn{4}{c}{ Ayamé } & \multicolumn{5}{c}{ Bakro } \\
\cline { 2 - 9 } & Min & Moy & Max & E-type & Min & Moy & Max & E-type \\
\hline $\mathrm{T}^{\circ}$ eau $\left({ }^{\circ} \mathrm{c}\right)$ & 28,4 & 28,8 & 29,6 & 0,4 & 26,1 & 28,2 & 29,3 & 1,1 \\
$\mathrm{pH}$ & 7,1 & 7,5 & 7,6 & 0,2 & 7,4 & 7,7 & 7,9 & 0,2 \\
$\mathrm{O}_{2}\left(\mathrm{mg} .1^{-1}\right)$ & 5,2 & 5,6 & 7,8 & 1,0 & 3,2 & 5,0 & 6,7 & 1,3 \\
Cond. $\left(\mu \mathrm{S} . \mathrm{cm}^{-1}\right)$ & 78,0 & 81,7 & 99,9 & 8,8 & 55,7 & 59,4 & 65,9 & 3,6 \\
Trans. $(\mathrm{m})$ & 1,1 & 1,3 & 1,6 & 0,3 & 1,0 & 1,1 & 1,3 & 0,1 \\
$\mathrm{N0}_{3}$-N $\left(\mathrm{mg} .1^{-1}\right)$ & 0,1 & 0,4 & 0,5 & 0,2 & 0,1 & 0,2 & 0,4 & 0,1 \\
$\mathrm{P0}_{4}$-P $\left(\mathrm{mg} \cdot 1^{-1}\right)$ & 0,8 & 1,6 & 1,9 & 0,5 & 0,5 & 0,7 & 0,9 & 0,1 \\
\hline
\end{tabular}


hétérogènes sur la colonne d'eau à Ayamé, avec des maximums d'activité photosynthétique en surface $(0 \mathrm{~m})$ et à $2 \mathrm{~m}$ de profondeur. Par contre à cette même période, les productions à Bakro baissent progressivement de la surface en profondeur. Contrairement aux mois précédents, de mars à mai, on observe une tendance à l'accroissement progressif de l'activité photosynthétique dans la colonne d'eau sur les deux stations. A Bakro, la teneur minimale journalière a été atteinte en mars dans la couche superficielle $(0 \mathrm{~m})$ et la valeur maximale relevée en avril à 2 mètres de profondeur. Ces valeurs extrêmes sont comprises entre 0,05 et $0,49 \mathrm{mg} \cdot \mathrm{I}^{-1} \cdot \mathrm{j}^{-1} \mathrm{de}$ carbone, mais la teneur moyenne pondérée sur la colonne d'eau (0-3 $\mathrm{m}$ ) ne dépasse pas 0,3 mg..$^{-1} \cdot j^{-1}$ de carbone. Les teneurs à Ayamé oscillent entre 0,19 en surface $(0 \mathrm{~m})$ en mars et $1,2 \mathrm{mg} \cdot{ }^{-1} \cdot \mathrm{j}^{-1}$ de carbone à $1 \mathrm{~m}$ de profondeur en décembre mais la valeur moyenne pondérée reste élevée $\left(0,56 \mathrm{mg} . \mathrm{I}^{-1} . \mathrm{j}^{-1}\right.$ de carbone), soit un peu plus du double de celle obtenue à Bakro $\left(0,25 \mathrm{mg} \cdot \mathrm{I}^{-1} \cdot \mathrm{j}^{-1}\right)$. Dans la zone euphotique étudiée, les activités photosynthétiques journalières par unité de surface (Tableau 3 ) sont de même ordre de grandeur dans les deux stations du lac. Elles varient de 0,18 à $0,35 \mathrm{~g} \mathrm{C} \cdot \mathrm{m}^{-2} \cdot \mathrm{j}^{-1}$ à Bakro et de 0,43 à $0,81 \mathrm{~g} \mathrm{C} \cdot \mathrm{m}^{-2} \cdot \mathrm{j}^{-1}$ à Ayamé. Bien que les productivités soient relativement plus faibles à Bakro qu'à Ayamé, aucune variation significative n'a été observée entre ces deux stations $(p>0,05)$. Par ailleurs, les résultats de la matrice de corrélation de Pearson (Tableau 4) montrent que les variables les plus corrélées à la productivité phytoplanctonique à chaque station sont les P04-P $(r>0,6$; $p>0,05)$ et N03-N $(r>0,8 ; p>0,05)$.

Tableau 2 : Production d'oxygène et assimilation du carbone au niveau des profils verticaux $0-3 \mathrm{~m}$ aux stations de Bakro et d'Ayamé.

Oxygen production and carbon assimilation within 0-3 $m$ vertical profiles at Bakro and Ayamé stations.

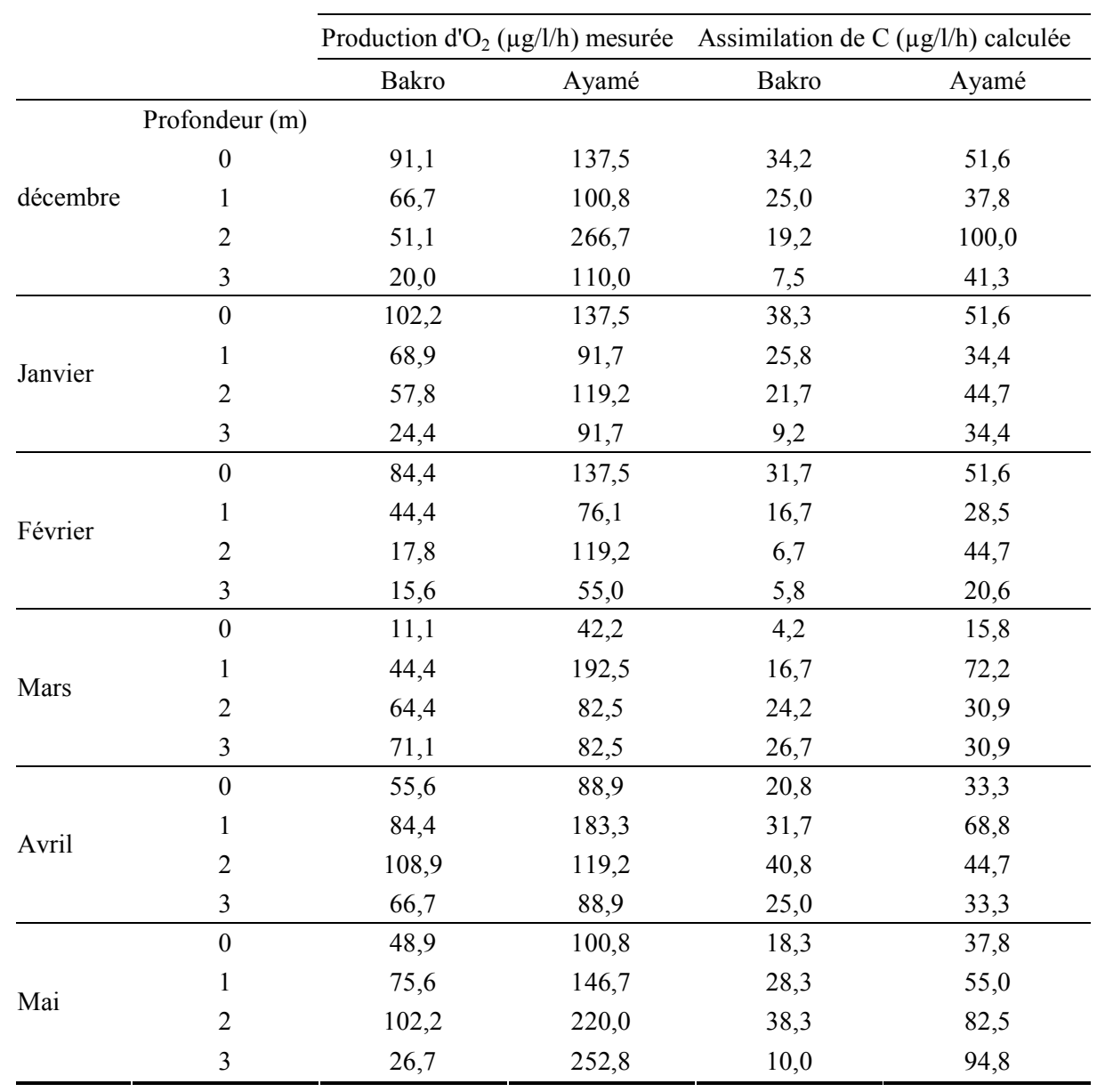


Taux de production primaire $(\mathrm{mg} \mathrm{C} / \mathrm{l} / \mathrm{j})$

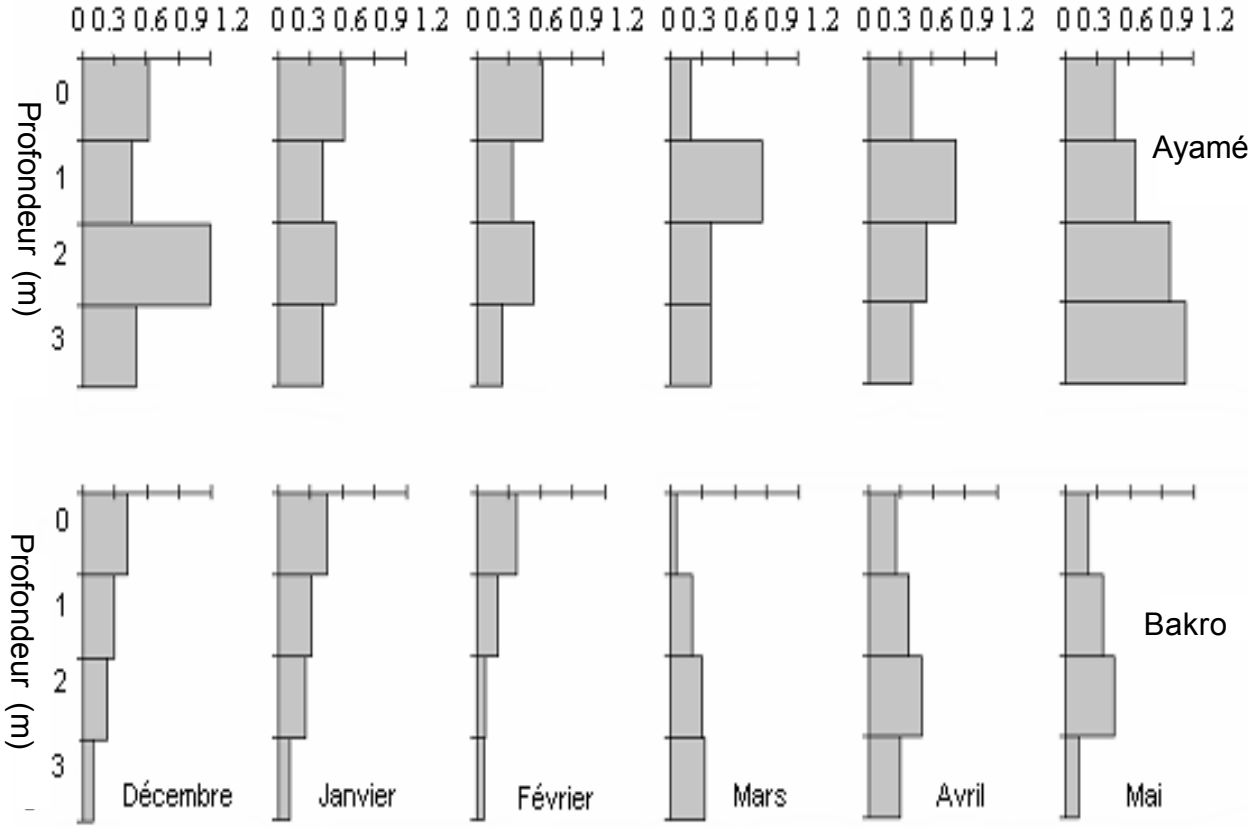

Figure 2 : Evolution des profils verticaux de production primaire dans la colonne d'eau aux stations de Bakro et d'Ayamé de décembre à mai.

Changes in vertical profiles of primary production in water column at Bakro and Ayamé stations from december to may.

Tableau 3 : Valeurs minimales (Min), maximales (Max) et moyennes (Moy) de la production primaire intégrée dans la zone euphotique à chaque station.

Minimum (Min), maximum (Max), means (Moy) values for primary production integrated into euphotic layer at each station.

\begin{tabular}{lcccc}
\hline \multirow{2}{*}{ Stations } & \multicolumn{4}{c}{ Production primaire en $\mathrm{g} \mathrm{C.} \mathrm{m}^{-2} \cdot \mathrm{j}^{-1}$} \\
\cline { 2 - 5 } & Min & Max & Moy & Ecart-type \\
\hline Bakro & 0,18 & 0,35 & 0,26 & 0,06 \\
Ayamé & 0,43 & 0,81 & 0,57 & 0,149 \\
\hline
\end{tabular}

Tableau 4 : Matrices de corrélations de Pearson pour la productivité phytoplanctonique et les variables physico-chimiques des stations Ayamé et Bakro.

Pearson's correlation matrix of phytoplanktonic produvtivity and physical and chemical variables at Ayamé and Bakro stations.

\begin{tabular}{llccccccc} 
& Stations & $\mathrm{T}^{\circ}$ eau & $\mathrm{pH}$ & $\mathrm{O}_{2}$ & Cond. & Trans. & $\mathrm{N0}_{3}-\mathrm{N}$ & $\mathrm{P0}_{4}-\mathrm{P}$ \\
\hline Productivité & Ayamé & $-0,19$ & 0,30 & 0,18 & 0,15 & 0,14 & $0,86 * * *$ & $0,62 *$ \\
phytoplanctonique & Bakro & 0,05 & $-0,20$ & 0,34 & $-0,48$ & 0,15 & $0,88 * * *$ & $0,94 * * *$ \\
\hline
\end{tabular}




\section{DISCUSSION}

L'évolution spatio-temporelle de la production primaire dans le lac d'Ayamé montre que la communauté phytoplanctonique présente différents stades de succession d'activité photosynthétique dans les deux stations échantillonnées. Les plus fortes productivités se situent d'avril à mai (période de début de saison de pluies). C'est également à cette période que les teneurs maximales en sels nutritifs $\left(\mathrm{PO}_{4}-\mathrm{P}\right.$, $\mathrm{NO}_{3}-\mathrm{N}$ ) ont été mesurées. La série de corrélations de Pearson impliquant l'ensemble des variables physico-chimiques, révèle une corrélation positive entre ces nutriments (nitrates, phosphates) et la productivité phytoplanctonique. La hausse des sels nutritifs en début de saison de pluies (avril-mai) pourrait résulter des apports en eau du bassin versant qui arrivent dans le lac, après avoir ruisselé sur les terres agricoles (Biggs, 2000 ; Ouattara et al., 2001). Les eaux du lac deviennent donc favorables à l'accroissement du phytoplancton en cette période.

En revanche, les productivités phytoplanctoniques sont moins importantes en janvier et février (saison sèche). Ceci pourrait s'expliquer par des teneurs peu élevées en sels nutritifs s'accompagnant d'une faible densité phytoplanctonique comme l'ont observés Ouattara et al. (2001). L'apport de ces nutriments dans la productivité phytoplanctonique a été mis en évidence par Arfi (2003) dans le réservoir de Sélingué au Mali. Par ailleurs, les variations de productivités phytoplanctoniques ne peuvent être interprétées seulement en termes de variabilité physico-chimique. En effet, selon Hart (2006) et Komárková (1998) la consommation du phytoplancton par les herbivores (crustacés planctoniques filtreurs et juvéniles de poissons) pourrait fortement influer sur les variations de ses populations. Les profils verticaux obtenus de mars à mai à Bakro sont d'allures très proches de ceux observés à Ayamé. Les algues sont en pleine activité en profondeur et en activité plus réduite au-dessus (Figure 2). Cette faible productivité de surface pourrait être due à la photo-inhibition. Cette observation a été faite par Dufour et Durand (1982) dans les systèmes lagunaires ivoiriens et par Lazzaro (1981) dans le lac Titicaca.

Par ailleurs, les fortes activités photosynthétiques mesurées à des profondeurs de 2 et 3 mètres, correspondent vraisemblablement à une accumulation des populations de phytoplancton physiologiquement actives. Cela peut s'expliquer par le brassage des eaux occasionnés par les vents ou par les mouvements de l'eau (Ouattara et al., 2001). Les mesures de productivité phytoplanctonique ont montré que les variations ne sont pas significatives entre les deux stations échantillonnées $(p>0,05)$. Par ailleurs, la productivité phytoplanctonique des deux stations du lac d'Ayamé I variant entre 0,18 et $0,81 \mathrm{~g} \mathrm{C} \cdot \mathrm{m}^{-2} \mathrm{j}^{-1}$ sont comparables à celles observées par Arfi et al. (2001) dans les petits barrages du nord de la Côte d'Ivoire $\left(<1 \mathrm{~g} \mathrm{C} . \mathrm{m}^{-}\right.$ $\left.{ }^{2} \cdot j^{-1}\right)$. Elles sont cependant inférieures à celles notées par Kassoum (1977) au lac Kossou $\left(<2,7 \mathrm{~g} \mathrm{C} \cdot \mathrm{m}^{-2} \mathrm{j}^{-1}\right)$ et par Patterson et Kachinjika (1995) au lac Malawi $\left(<1,5 \mathrm{~g} \mathrm{C} \cdot \mathrm{m}^{-2} \cdot \mathrm{j}^{-1}\right)$. En rapportant ces variations journalières à une année, la productivité du lac d'Ayamé I pourrait varier entre 65,7 et $295,6 \mathrm{~g} \mathrm{C} . \mathrm{m}^{-2}$.an-1. Au regard du niveau de productivité phytoplanctonique, un tel lac pourrait être rangé, selon Mason (1991), parmi les lacs mésotrophes.

\section{CONCLUSION}

Cette étude a permis de suivre l'évolution spatiotemporelle de la production primaire liée à l'activité photosynthétique du phytoplancton dans le barrage hydroélectrique d'Ayamé I. Les productivités mesurées sont plus fortes en début de saison de pluies qu'en saison sèche. Les teneurs en nitrates et phosphates sont corrélées aux productivités dans chaque station étudiée. Ces résultats montrent que les nutriments jouent un rôle déterminant sur la productivité phytoplanctonique et expliquent les variations temporelles observées à Bakro et Ayamé au cours de la période d'étude. Par ailleurs, il n'apparaît pas de différences notables de productivité phytoplanctonique d'une station à l'autre. Compte tenu des valeurs de productivités obtenues, le lac d'Ayamé I présente les caractéristiques d'un lac mésotrophe.

\section{REMERCIEMENTS}

Nous tenons à remercier les membres $d u$ projet VLIR pour leur aide et leur grande efficacité. Nos remerciements s'adressent également à la Coopération Technique Belge 
(ex-AGCD) pour le soutien financier de cette étude.

\section{REFERENCES}

Arfi R., Bouvy M., Cecchi P., Pagano M., Saint-Jean L. and S. Thomas. 2001. Factors limiting phytoplankton productivity in 49 shallow reservoirs of North Côte d'Ivoire (West Africa). Aquatic Ecosystem Health and Management $4: 123$ - 138.

Arfi R. 2003. The effects of climate and hydrology on the trophic status of Sélingué Reservoir, Mali, West Africa. Lakes and Reservoirs : Research and Management $8(3-4):$ 247-257.

Biggs B. J. F. 2000. Eutrophication of streams and rivers: dissolved nutrient-chlorophyll relationship for benthic algae. J. N. Am. Benthol. Soc. 19: 17 - 31 .

Biro P. and L. Voros. 1990. Trophic relationship between primary producers and fishyiels in lake Balaton. Hydrobiologia 191: 209 - 220.

Cyr H. and R. H. Peters. 1996. Biomass-size spectra and the prediction of fish biomass in lakes. Can J. Fish. Aquat. Sci. 53 : 994 - 1006.

Degnbol P. and S. Mapila. 1982. Limnological studies on the pelagic zone of lake Malawi from 1978-1981. FAO Technical Report. $1: 3-48$.

Downing J. A., Plante C. and S. Lalond. 1990. Fish production correlated with primary productivity, not the morphoedaphic index. Can J. Fish. Aquat. Sci. 47 : 1929 - 1936.

Dufour P. et J. R. Durand. 1982. La production végétale des lagunes de Côte d'Ivoire. Rev. Hydrobiol. trop. 15 (3) : 209 - 230.

Ganf G. G. 1975. Photosynthetic production and irradiance photosynthesis relationship of phytoplankton from a shallow equatorial lake (Lake George). Oecologia 18 : $165-183$

Gourène G., Teugels G. G., Hugueny B. et D. F. E. Thys Van Den Audenaerde. 1999. Evaluation de la diversité ichtyologique d'un bassin ouest africain après la construction d'un barrage. Cybium 23 (2) : 147 - 160.

Hart R. C. 2006. Phytoplankton dynamics and periodicity in two cascading warm-water reservoirs from 1989 to 1997 - taxonomic and functional (C-S-R) patterns, and determining factors. Water SA 32 (1) : 81 - 92.

Kassoum T. 1977. Premières données sur les facteurs du milieu et sur la production primaire du lac Kossou. Thèse de doctorat $3^{\text {e }}$ cycle, Université Nationale d'Abidjan (Côte d'Ivoire), $106 \mathrm{p}$

Komárková J. 1998. Fish stock as a variable modifying trophic pattern of phytoplankton. Hydrobiologia 369 - 370 : 139 - 152.

Lazzaro X. 1981. Biomasses, peuplements phytoplanctoniques et production primaire du lac Titicaca. Rev. Hydrobiol. trop. 14 (4) : $349-380$

Mason C. F. 1991. Biology of freshwater pollution. Second edition, Longman Scientific and Technical, New York, 350 p.

Melack J. M. 1976. Primary productivity and fish yields in tropical lakes. Trans. Am. Fish. Soc. $105: 575$ - 580 .

Oglesby R. T. 1977. Relationship of fish yield to lake phytoplankton standing crop, production and morphoedaphic factors. J. Fish. Res. Board Can. 34 : $2271-2279$

Ouattara A., Podoor N. et G. Gourène. 2001. Etudes préliminaires de la distribution spatiotemporelle du phytoplancton dans un système fluvio-lacustre africain (bassin Bia, Côte d'Ivoire). Hydroécol. Appl. 13 (1) : 113 - 132

Patterson G. and O. Kachinjika. 1995. Limnology and phytoplankton ecology. In : A. Menz (Ed.). The fishery potential and productivity of the pelagic zone of lake Malawi/Niassa Natural resources Institute, Chatham, United Kingdom : pp 1 - 67.

Vaquer A., Pons V. et J. Lautier. 1997. Distribution spatio-temporelle du phytoplancton dans le réservoir de Petit-Saut (Guyane Française). Hydroécol. Appl. 9 (1-2) : $169-193$

Vollenweider R. A. 1974. A manual on methods for measuring primary production in aquatic environments, IBP Handbook $n^{\circ} 12$, Second edition. Blackwell Scientific publications, $225 \mathrm{p}$.

Wetzel R. G. and G. E. Likens. 1995. Limnological analysis. Second edition. Springer - Verlag, $387 \mathrm{p}$. 